Article

\title{
Enabling Large-Scale IoT-Based Services through Elastic Publish/Subscribe
}

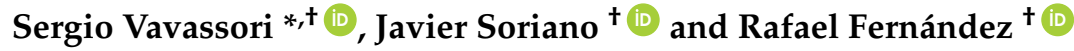 \\ School of Computer Science, Universidad Politécnica de Madrid, 28660-Boadilla del Monte, Madrid, Spain; \\ jsoriano@fi.upm.es (J.S.); rfernandez@fi.upm.es (R.F.) \\ * Correspondence: svavassori@fi.upm.es \\ + These authors contributed equally to this work.
}

Received: 1 August 2017; Accepted: 14 September 2017; Published: 19 September 2017

\begin{abstract}
In this paper, we report an algorithm that is designed to leverage the cloud as infrastructure to support Internet of Things (IoT) by elastically scaling in/out so that IoT-based service users never stop receiving sensors' data. This algorithm is able to provide an uninterrupted service to end users even during the scaling operation since its internal state repartitioning is transparent for publishers or subscribers; its scaling operation is time-bounded and depends only on the dimension of the state partitions to be transmitted to the different nodes. We describe its implementation in E-SilboPS, an elastic content-based publish/subscribe (CBPS) system specifically designed to support context-aware sensing and communication in IoT-based services. E-SilboPS is a key internal asset of the FIWARE IoT services enablement platform, which offers an architecture of components specifically designed to capture data from, or act upon, IoT devices as easily as reading/changing the value of attributes linked to context entities. In addition, we discuss the quantitative measurements used to evaluate the scale-out process, as well as the results of this evaluation. This new feature rounds out the context-aware content-based features of E-SilboPS by providing, for example, the necessary middleware for constructing dashboards and monitoring panels that are capable of dynamically changing queries and continuously handling data in IoT-based services.
\end{abstract}

Keywords: content-based publish/subscribe; Chandy-Lamport algorithm; distributed snapshot; distributed system; Internet of Things

\section{Introduction}

The Internet of Things (IoT) relies on a continuously growing number of interconnected uniquely addressable heterogeneous electronics (UAHE), including sensors, actuators, smart devices, embedded computers, etc., producing tremendous amount of data about the surrounding living environments to nourish an ever-growing number of services [1]. The large-scale nature of IoT-based services can be effectively and efficiently facilitated and supported via utilizing Cloud Computing infrastructures and platforms for providing flexible and extensive computational power, resource virtualization and high-capacity storage for data streams. Data brokerage [2] is a key concept for handling such a massive number of IoT-based services in a cloud environment. Since cloud environments are intrinsically dynamic, the same brokerage system has to handle variability, scale out to cope with new load, and scale in when the peak has passed in order to save resources. However, today's cloud systems, like OpenStack [3] or OpenNebula [4], offer no more than a queuing system for delivering notifications from components or at best a topic-based publish/subscribe [5-7]. The same thing happens with publish/subscribe services available online like Amazon Web Services (AWS) Simple Notification Service [8], Google Cloud Pub/Sub [9] or Microsoft Azure Service Bus [10]. This is a limiting factor since it requires queue and topic management: publishers must decide in which queue or topic to publish their messages and interested components must decide from which queue they read. 
Content-based publish/subscribe systems (CBPS) [11,12] use dynamic message routing to avoid this limitation and are able to minimize message traffic through the distribution network.

Nonetheless, the classical network of brokers that made up the CBPS distribution network has some intrinsic scalability limits: subscriptions must be propagated to all brokers and the number of connections to the system is dependent on the number of brokers. Two different strategies have been developed so far in order to solve the first limitation: covering and merging subscriptions and using advertisements to avoid sending subscriptions if there are no matching publishers. Covering and merging are, however, computationally expensive and only provide a real gain for heavily colliding distributions. On the other hand, advertisements provide the specific and definite benefit of reducing subscription dissemination, even though computation will increase slightly in order to perform routing $[11,13]$.

To overcome the second limitation, that is, the number of connections that the system can handle, the connection handling part needs to be decoupled from the routing part. Some systems like $[14,15]$ or others that use a distributed hash table (DHT) architecture for matchers [16] provide this feature; however, most require complex coordination to provide an elastic service or simply rely on the hypervisor layer to perform live migration [17].

We used the CBPS model provided by SilboPS [18], which already provided a solution to efficiently share and use sensor data coming from ubiquitous Wireless Sensor Networks (WSN) across a plethora of applications, in order to implement an elastic version of it, called E-SilboPS. Support for dynamic state partitioning was required to properly deliver elasticity, and this meant that we had to restructure the internal architecture. Nonetheless, the Application Programming Interface (API) and message compatibility have been conserved to guarantee transparent substitution from the standard to the elastic version.

Our proposal allows for the provisioning of an elastic CBPS system capable of providing large-scale IoT environments with an uninterrupted service even during the scaling operation, which is a requirement for today's large-scale IoT based Services involving billions of messages gathered from an ever-growing number of sensors.

The remainder of the paper is organized as follows: Section 2 shows the general architecture of the system and a brief review of the related work is presented in Section 3, highlighting the differences of our research with respect to other noteworthy systems; Section 4 describes the specific architecture of E-SilboPS, whereas Section 5 explains in details how the scaling algorithm works using a scale-out use case. Then, Section 6 analyzes the performances of E-SilboPS executing a scale-out with different configurations, detailing throughput, speedup and efficiency for each configuration in order to illustrate its strengths and the best deployment for a given load. Finally, we state our future goals and highlight the findings of this research in Sections 7 and 8.

\section{General Architecture}

Connecting objects or things involves the need to overcome a set of problems arising in the different layers of the communication model. Using their data or acting upon them requires interaction with an ever-growing, heterogenous environment of devices running different protocols, due to the lack of globally accepted standards, dispersed and accessible through multiple wireless technologies. For this reason, approaching an IoT platform must enable intermediation, data gathering and data publishing functions.

FIWARE IoT services enablement platform [19] offers an architecture of components (called Generic Enablers or GEs) specifically designed to face these problems by allowing to capture data from, or act upon, IoT devices as easily as reading/changing the value of attributes linked to context entities. Figure 1 depicts the general architecture of this platform, which includes:

- The FIWARE backend IoT Device Management GE enables creation and configuration of IoT Agents (left part of Figure 1) that connect to sensor networks (ETSI M2M, MQTT, IETF CoAP, etc). IoT Agents solve the issues of heterogeneous environments where devices with different 
protocols approach to a common data format. IoT Agents act as mediators/translators between the protocols that devices use to send or receive information (HTTP Ultralight, MQTT, OMA Lightweight M2M), and a common language and data model across all the platform: FIWARE Next Generation Service Interfaces (NGSI) [20].

- The FIWARE Context Broker GE (center) to gather, publish, query and subscribe-to in-time IoT-enabled context information at large scale, in order to be used by hosted applications. The Context Broker implements the OMA NGSI-9/10 API [21] and it relays internally on the E-SilboPS component for all the publish/subscribe communication features. E-SilboPS provides the Context Broker with an elastic content-base publish/subscribe (CBPS) system specifically designed to support context-aware sensing and communication in IoT-based services.

FIWARE NGSI brings a simple yet powerful RESTful API enabling access to IoT-enabled context information. It is intended to manage the entire lifecycle of context information, including updates, queries, registrations, and subscriptions. Managing this information is possible since Context Broker keeps virtual representations of the physical devices. Interaction with devices will happen by updating and modifying the virtual representations attached/corresponding to them. From an architectural point of view, the Context Broker acts as a blackboard in a typical blackboard architecture. It is the core and control piece of the platform, in charge of interacting with the other components and agglutinate data.

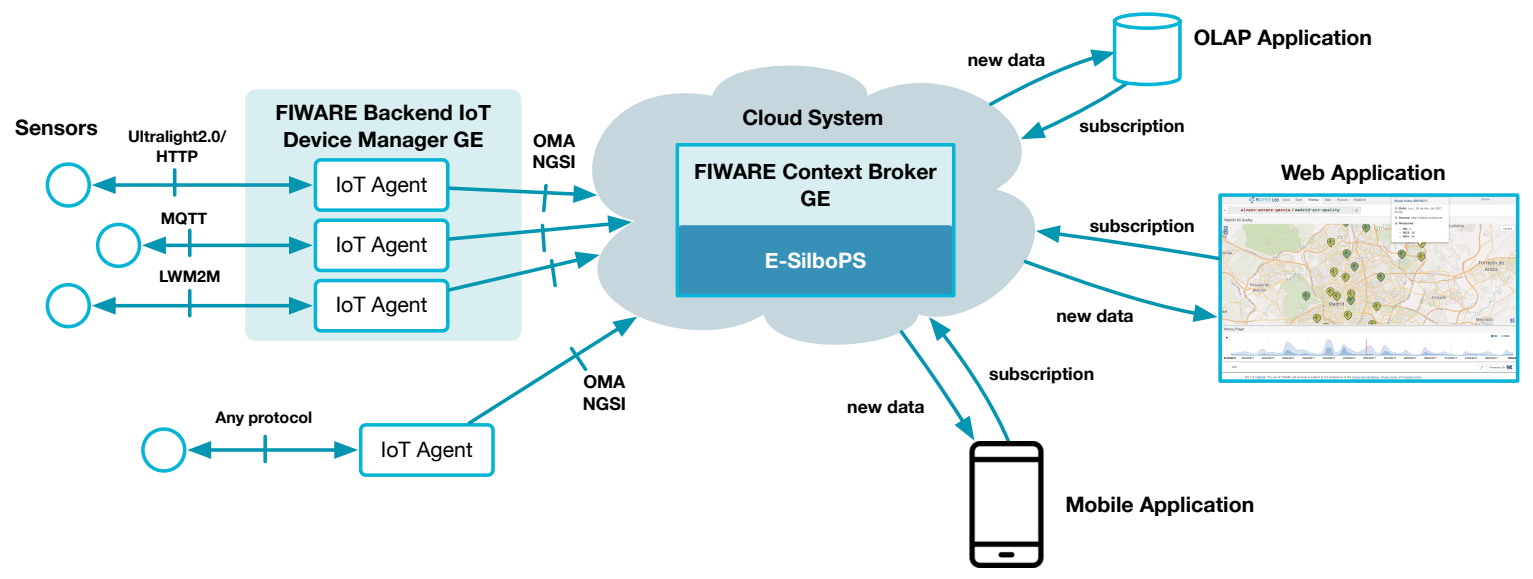

Figure 1. General architecture to support large scale IoT services. IoT sensors (left) are sending their data to the Context Broker and this one leverages E-SilboPS to relay messages to the interested subscribers (right).

By using this architecture, as shown in Figure 1, IoT devices will be represented in a FIWARE platform as NGSI entities in the Context Broker. This means that you can query or subscribe to changes of device parameters status by querying or subscribing to the corresponding NGSI entity attributes at the Context Broker. E-SilboPS represents a key internal platform asset to achieve elasticity in the distribution of context information mediated by the Context Broker in a cloud-enabled environment.

Subscribers can be whatever application or user is interested in the data and do not need to know where the actual sensor is or the specific format it uses to send its data, thanks to the common NGSI API. An example of those can be smartphones applications to allow mobile users to receive important updates, web application for a wider analysis or data-storage facility to perform On Line Analytical Processing (OLAP) or other data-intensive tasks (right part of) Figure 1.

For the sake of clarity and for evaluation purposes, we use a running IoT example based on the air-pollution station deployed in the city of Madrid. In our example, we have gathered air pollution data generated from sensors mounted in fixed station (the left part of Figure 1), published to the Context Broker (center) and, by using specific subscriptions, consuming the new data (right) by 
displaying them in a web portal [22] to show real-time as well as historical time series categorized by pollutant, as shown in Figure 2.

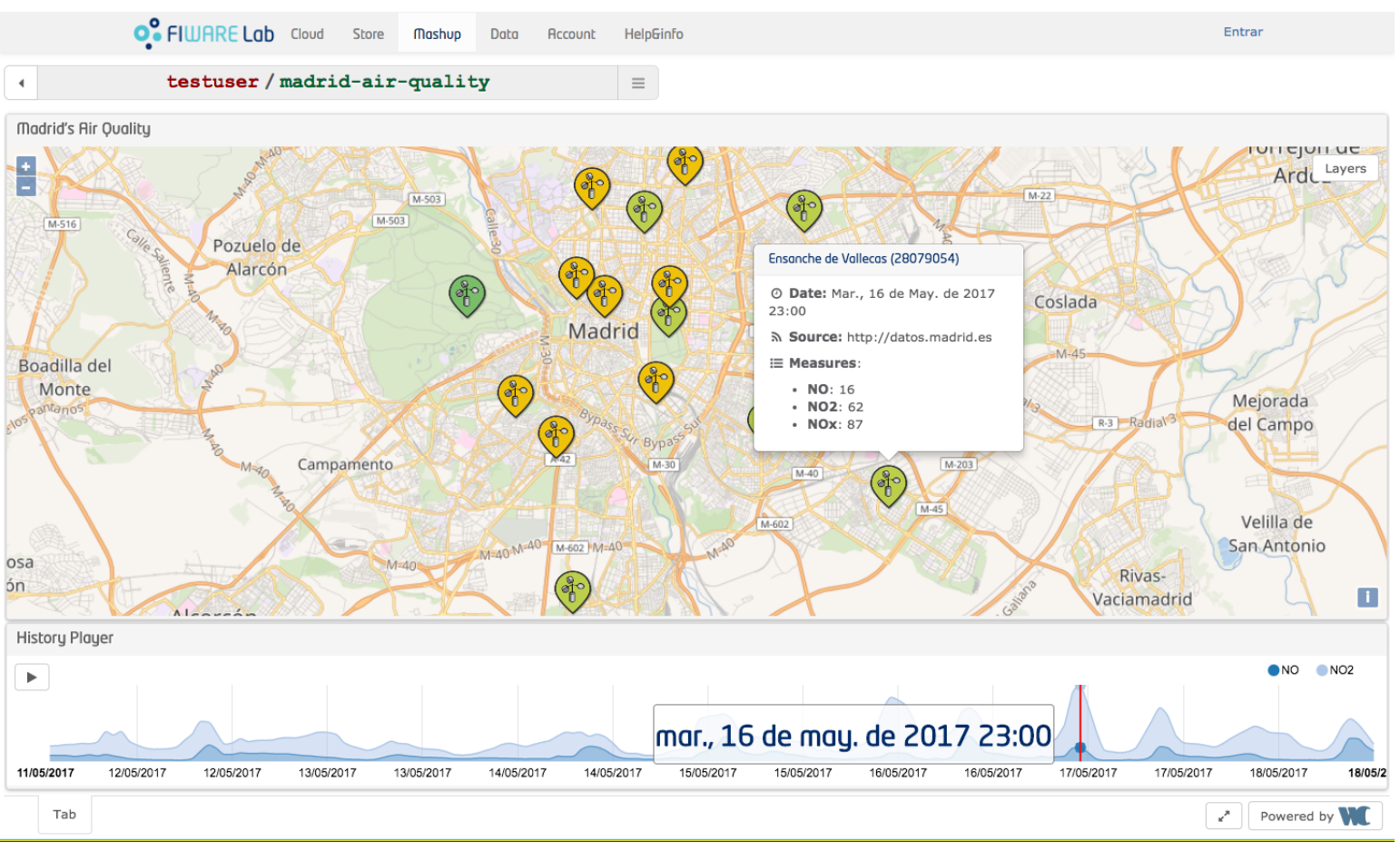

2017 IWARE. The use of FIWARE Lab services is subject to the acceptance of the Terms and Conditions, privacy Policy and Cookies Policy.

Figure 2. An example of subscriber application: the interactive map shows the various real-time contaminants measurements done by pollution station in the city of Madrid.

\section{Related Work}

In the context of WSN, the adoption of decentralized publish-subscribe services is already established. These approaches are complementary to our work and can use our system as a way to connect various IoT services. For example, the work in [23] focuses on the internal WSN routing instead of the message distribution between IoT services, so it could benefit from using our solution to get connected to third party services.

Many elastic CBPS have been proposed over the last few years. However, most offer automatic scalability rather than proper elasticity. As a matter of fact, elasticity is the degree to which a system is able to adapt to workload changes by autonomously provisioning and deprovisioning resources to assure that the available resources match the current demand as closely as possible at any time [24]. This means that the application of the new configuration should not affect the offered service or require a restart of the system that always amounts to downtime.

The most noteworthy examples of systems are BlueDove, SEMAS and E-StreamHub [15-17]. BlueDove and SEMAS use a fat Access Point layer that handles the mechanism for selecting which matcher holds the subscriptions whose attributes are compatible with the current notifications, whereas matchers are organized in a P2P structure, typically using a DHT like Chord [25]. This approach is capable of selecting only the matchers that are of potential interest and it does not waste the time of others that already know that they have no subscriptions matching the notification. However, it requires some state duplication between the Access Point layer and the Matcher layer. Additionally, the fact that the access points include the state makes the scaling algorithm more complex since both layers have to be updated consistently.

On the other hand, E-StreamHub is the elastic version of Streamhub [14]. Streamhub has a cleaner architecture that is able to add and remove operators from each layer in a simpler manner. It also has 
stateless access points, and thus requires less complex algorithms to perform a consistent change view. In addition, the Matcher layer does not use a DHT, and it is a single hop from the Access Point layer, thereby reducing matching latency. Due to its implementation, however, it does no dynamic state partitioning; instead, it partitions into slices its internal state during startup and then moves the slices from one Virtual Machine (VM) to another in order to scale out.

We argue that this approach does not provide proper elasticity since it limits a priori the maximum achievable scale-out on the grounds not of zero efficiency [26] or a slowdown of the speedup but simply because the partitioning parameter is wrongly forecast during startup. Irrespective of the chosen partitioning parameter value, the system will have an intrinsic avoidable overhead for all less-demanding workload, as well as a fixed upper bound for scalability. This goes completely against the definition given by [24].

As a matter of fact, this strategy can be seen as an application-specific version of VM live migration already offered by today's hypervisors like KVM, Xen, VirtualBox or VMWare. Application-agnostic hypervisor-based VM live migration is more valuable in the general case, but it has the same limitation as E-StreamHub, specifically with respect to dynamic state partitioning. In Section 4, we show how E-SilboPS can overcome the above weakness, while retaining the cleaner and simpler architecture of E-StreamHub.

\section{Concept}

The system should have a global elasticity property in order to overcome the weakness of sizing a scalable monitoring system with an a priori deployment, namely wasting resources or underperforming monitoring because of the changing load. In our approach, we have added this property to all operators. In this way, we can adapt each layer to the specific environment without worrying about providing a setup that fits from the start. In addition, temporary deviations from the standard usage pattern, like load spikes, can be handled without having to restart the system.

E-SilboPS is divided into four layers, as shown in Figure 3, similar to the engine described in $[14,17]$, and each operator layer can scale in and out independently of the others. Some orchestration between layers is needed, however, to maintain state consistency, and it is done by creating and removing nodes from the Distributed Coordinator (DC) in order to fire events to the interested layers; the DC is implemented using Zookeeper (We use ZooKeeper to ensure a reliable distributed coordination between operator slices. [27]) These four layers are:

Access Point (AP) dispatches incoming subscriptions to the correct matcher and broadcasts notifications to all matchers.

Matchers (M) store incoming subscriptions in the internal structure, match notifications against subscriptions, and dispatch the local result set to the Exit Point layer.

Exit Point (EP) collects all partial results sets coming from the Matcher layer, which it merges in the final results set and then sends the notification to all Connection Points found in this set.

Connection Point (CP) represents the system entry and exit point; it handles the connection with clients and sends the received notifications to the interested subscribers; it forwards subscriber subscriptions and publisher notifications to the Access Point.

To perform proper scaling $[24,28]$, the system must be able to match the variable demand of workload and, at the same time, control the cost of the deployed infrastructure. Our solution uses the Chandy-Lamport algorithm [29], also known as the snapshot algorithm, to create a consistent cut of the global state of the system. It then executes the algorithm that we designed to transfer the state throughout the system, repartitioning it according to the new available slices. One noteworthy feature of our algorithm is that it is capable of matching notifications while restructuring its internal state and has a very short no-matching time before switching to the new state. 


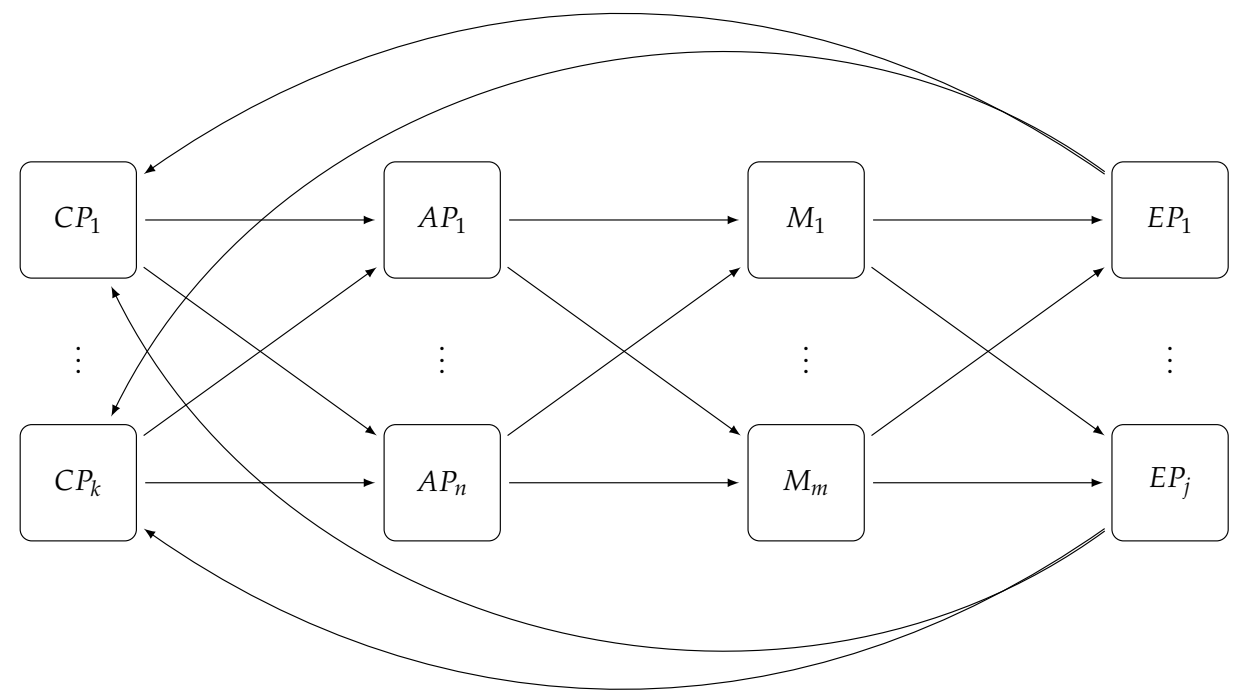

Figure 3. E-SilboPS architecture for the all layers: Connection Point, Access Point, Matcher and Exit Point. Notifications and subscriptions are coming from the Connection Point layer to the Access Point layer in order to be dispatched to the correct Matcher layer instance $\left(M_{1} \ldots M_{n}\right)$ and then it will be sent to a specific Exit Point instance to collect all responses and avoid message duplication. Finally, the message will be sent to the selected Connection Point to be delivered to clients. In this figure, the Distributed Coordinator, since it has a direct connection to each slice of each layer, has been omitted in order to keep the diagram clean.

\section{The Algorithm}

In this section, we explain our scaling algorithm: there are three possible layer reconfigurations, one for each operator except for Connection Point. Coordination with other layers may be required depending on the layer and operator state. For each kind of reconfiguration, we will list the operations to be done by each layer in order to keep a consistent state.

\subsection{Access Point Scaling}

Access point scaling is straightforward since it is a stateless operator, and no coordination is required because it is usually bound with a Connection Point. This means that layer reconfiguration is limited to adding or removing target operators and opening or closing connections to the Matcher layer.

Access Point layer: start or stop instances according to the new configuration.

Matcher layer: no action.

Exit Point layer: no action.

\subsection{Matcher Scaling}

As shown in Figure 4, the scaling of the Matcher layer requires coordination with the other two layers since Access Point will need to use a new selector and buffer subscriptions and notifications, whereas Exit Point will have to change their internal state to correctly collect notifications coming from the new Matcher configuration. 


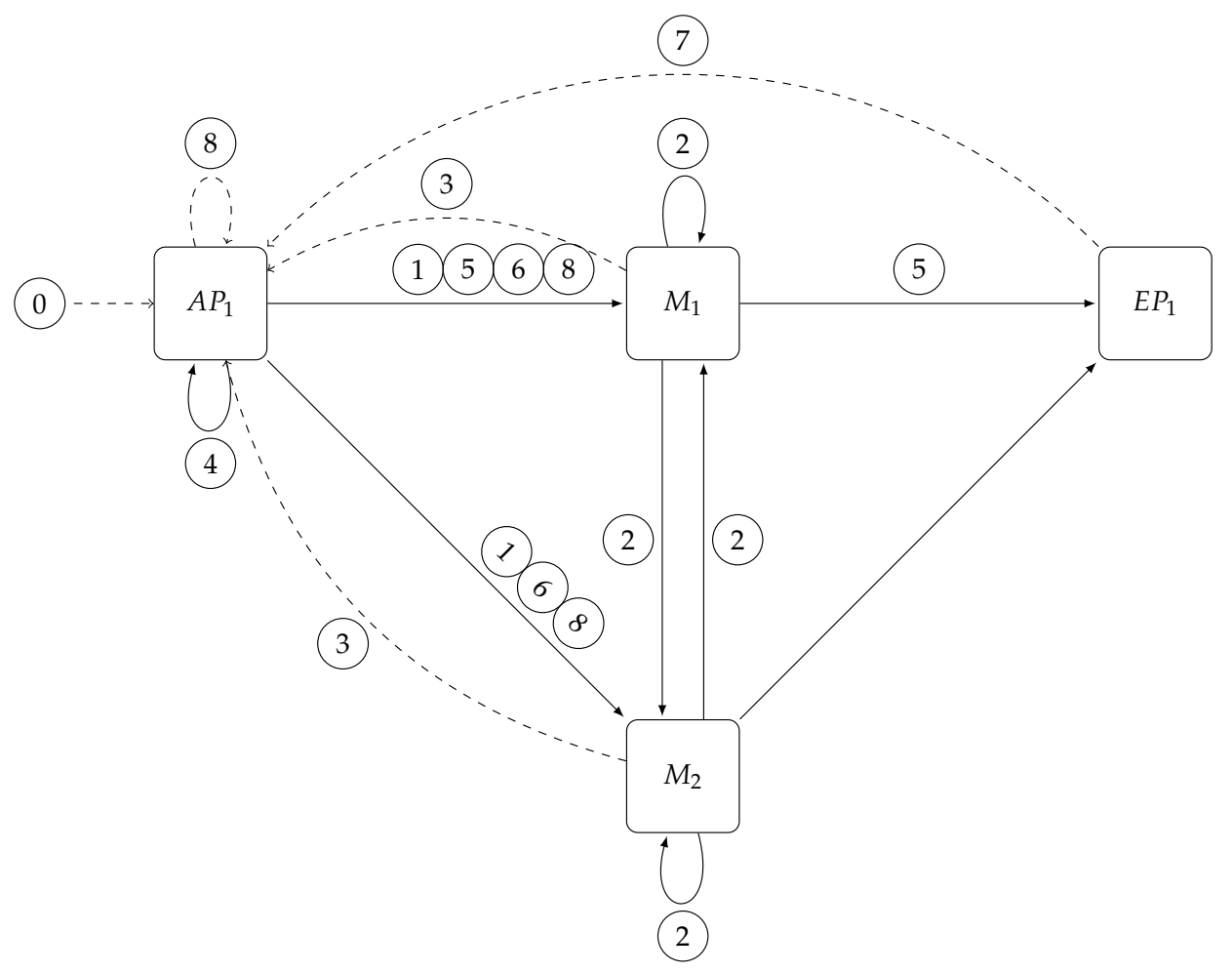

Figure 4. Messages exchanged during Matcher layer scale-out: solid lines represent connections between operators used by the system to send notifications and subscriptions. Dashed lines represent Distributed Coordinator-mediated messages used to synchronize layers, typically to unlock all operators from a layer waiting for another layer to complete its task.

The scaling process starts by sending a Start Scale-In/Out message to all Access Points (0); upon receiving this message each access point starts buffering subscriptions and forwards the same message, together with the new selector that they have to use, to all existing matchers (1). This renders the state of the Matcher layer temporarily unchangeable, although, at the same time, it is still able to process notifications. Thus, this phase is transparent for publishers, and subscribers will still receive notifications as usual, although they will not see any updates of the subscriptions that have changed in the meantime.

As soon as a matcher receives the first Start Scale-In/Out message, it connects to all matchers of the new configuration and partitions its internal state using the selector included in the received message. It then sends this new state to the respective matchers, itself included (2). In this way, all matchers know in advance from which matcher they will receive the new state, that is, from the matchers present in the new configuration in the event of a scale-out or from the matchers in the old configuration in the event of a scale-in.

When a matcher has received all state information, it sends a Change-Ready message to the Distributed Coordinator to notify the Access Point layer (3). This layer will be unlocked with a Change-Matcher-Ready when all matchers have finished (4). The Change-Matcher-Ready message signals to the Access Point layer to enqueue notifications and to send a Change-Matcher-On-Exit Point message to all old matchers (5) and a Change-Matcher to all (old+new) matchers (6) so that they can swap their internal state for the new one.

The Change-Matcher-On-Exit Point message must precede Change-Matcher because it is forwarded upon arrival at the Matcher layer to the Exit Point layer. This creates a consistent change of view of the Matcher layer state from the Exit Point layer perspective without direct synchronization between matchers. This message must be sent to all matchers in order to prevent subsequent notifications reaching a newly added matcher and being processed faster than the last notification sent 
before the state change. Otherwise, the exit point would associate this new notification with the wrong number of matchers, which, in the event of scale-out, would lead to the received subset of subscribers being notified too early. This would leave in starvation the remaining set of subscribers at the same exit point. In the event of scale-in, the notification will never be delivered to subscribers because the exit point will not receive all the expected sets for this purpose.

When an exit point receives the Change-Matcher-On-Exit Point message, it reads the current number of matchers from Distributed Coordinator and notifies its new state. The number read from the configuration will be immediately used as the new threshold before pushing notifications to subscribers.

Once all exit points have been updated, the Distributed Coordinator will send an Exit Point-Matcher-Updated to Access Point layer (7) to flush buffered notifications if scaling-out and wait for all access points to finish the operation. It will then send a Scale-End message to the Access Point layer 8, which will be forwarded to the Matcher layer and will close all unnecessary connections. On receiving the Scale-End message, each matcher will close its connections to others but will not forward this message to the Exit Point layer since no operation is needed for this purpose.

\section{Access Point layer}

- When receiving a Start Scale-In/Out message with a new matcher selector:

1. Continue to broadcast notifications to all current matchers

2. Buffer subscriptions in a queue

3. Send Start Scale-In/Out message to all (old+new) matchers with the new matcher selector to be used

- When receiving a Change-Ready message:

1. If in the scale-out phase, the notifications are no longer sent. (The notifications sent after change view could enter the new matcher instance, being matched sooner than other notifications from other matchers and reaching the Exit Point before the last notification of the previous group.)

2. The Change-Matcher-On-Exit Point message is sent to all old matchers

3. The Change-Matcher-Ready is sent to all (old+new) matchers

4. The current selector is exchanged for the new selector received from Access Point

5. While waiting Exit Point Matcher-Updated sends enqueued subscriptions to matchers

- When receiving a Exit Point Matcher-Updated message:

1. If it is in scale-out phase, flush enqueued notifications

2. Resume sending notifications

3. Signal Scale-End to the Distributed Coordinator

\section{Matcher layer}

- When the Matcher has received a Start Scale-In/Out message with a new matcher selector from all Access Points:

1. Notifications continue to pass through the matcher using current matching algorithm (No subscriptions can arrive because they are buffered at Access Point layer.)

2. Snapshot matcher's Subscription Map

3. Map snapshotted subscriptions set with new matcher selector and store the result in Map<Slice, Map<ConnectionID, Set<Subscription»>

4. Send the new mapping to each matcher and to itself 
- When receiving a Add-Matcher-State message:

1. The subscription set is added to the new matcher state

2. When all Add-Matcher-State messages have been received, the Change-Ready message is sent to Access Point through the Distributed Coordinator

- When receiving Change-Matcher-On-Exit Point:

1. Forward it to all Exit Points

- When receiving a Change-Matcher-Ready message:

1. Swap matcher state for new one

2. Start processing notifications and subscriptions

\section{Exit Point layer}

- When receiving a Change-Matcher-On-Exit Point message:

1. The new number of matchers is read from the DC and used as the current limit for notifications

2. Exit Point-Changed is sent to DC

\subsection{Exit Point Scaling}

Exit Point layer scaling (see Figure 5) has to be coordinated with the Matcher layer because a consistent cut of notification must be done. Even though no direct mechanism of synchronization between matchers is required, each matcher has to perform a set of operations such that the added (or removed) exit point instances will be used only after a consistent change view.

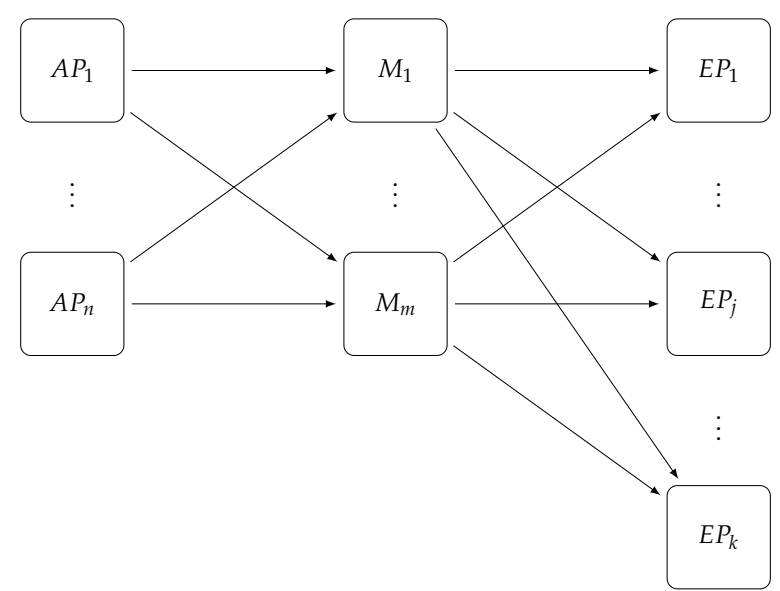

Figure 5. Exit Point layer scale-out: exit points from $j+1$ to $k$ are added to the current configuration.

An Exit-Point-Scaling message is first sent to access points which they then broadcast to matchers. The matchers use this message to create the new exit point selector and buffer notifications on an access point basis. Once it has received the respective message from all access points, the matcher processes all buffered notifications using the new exit point selector and sends a message to the Distributed Coordinator to notify its reconfiguration. After collecting all reconfiguration messages from matchers, the Distributed Coordinator sends a Scale-End message to the Access Point layer to clean up connections in the same manner as for Matcher layer scaling.

As shown in Figure 6, $A P_{1}$ sends notifications $N_{1}, N_{3}$ and $N_{5}$, whereas $A P_{2}$ sends notifications $N_{2}$ and $N_{4}$. However, due to the independent FIFO channels that connect access points to matchers, 
the order of delivery viewed by $M_{1}$ is $N_{1}, N_{3}$ and $N_{2}$, as opposed to $N_{2}, N_{1}$ and $N_{3}$, as viewed by $M_{m}$, before reception of the two Exit Point-Scaling messages. In addition, $M_{m}$ receives $N_{4}$ before $E P S_{1}$, but it knows that this notification will be in the Post set because it has already received $E P S_{n}$ from the same channel.

This proves that, even if the order of the messages received by matchers differs, their Pre and Post sets contain the same elements, so they can process notifications according to a consistent cut. In addition, the relative ordering of notifications coming from each access point is preserved, so, from a publisher's point of view, no out-of-order matching is performed.

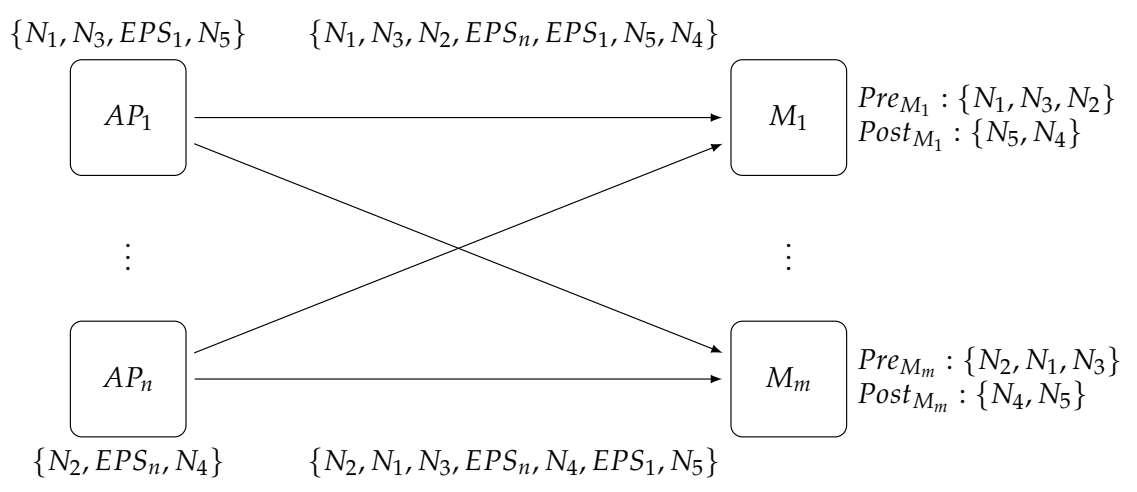

Figure 6. Matcher layer notifications cut: $A P_{1}$ sends odd notifications $\left(N_{1} \ldots N_{5}\right)$, whereas $A P_{n}$ sends even notifications $\left(N_{2} \ldots N_{4}\right) . E P S_{1}$ and $E P S_{n}$ represent the Exit Point-Scaling messages broadcast by each access point to all matchers.

\section{Access Point layer}

- When receiving Exit Point-Scaling:

1. Forward Exit Point-Scaling to all matchers

\section{Matcher layer}

- When receiving first Exit Point-Scaling:

1. Read from the DC the number of Access Point and Exit Point instances

2. Create the new Exit Point selector

3. Start enqueueing notifications from that Access Point slice

- When receiving subsequent Exit Point-Scaling:

1. Notifications from that Access Point slice are enqueued

- When receiving last Exit Point-Scaling:

1. The new Exit Point selector is adopted and all enqueued notifications are flushed

2. DONE message is sent to the DC to signal the end of current matcher reconfiguration

\section{Exit Point layer}

- Instances are added or removed according to the new configuration

\section{Evaluation}

To evaluate how our architecture will operate and deliver messages coming from IoT sensors to all the interested subscribers, we implemented E-SilboPS. 
E-SilboPS is the elastic version derived from SilboPS [30], our Java implementation of SIENA $[13,18]$ with improved context management. As usual for P/S systems, our software does not support transactional messages, but offers FIFO channels with reliable delivery. This implies that no message loss will be observed, unless there is a link failure, of which both channel endpoints are aware. Messages are processed in the same order as they arrive at the operator instances irrespective of their sending time. The software is available for research purpose upon request.

We ran our experiment on an Intel i7 $860 @ 2.80 \mathrm{GHz}$ machine equipped with $12 \mathrm{GiB}$ of RAM running Linux 4.2 and OpenJDK 7u91.

Unfortunately, we cannot compare directly E-SilboPS against E-StreamHub for two reasons: first, in their article, they wrote only the matching fraction of subscription per each notification, but there is no information about the distribution of the attributes to create those notifications and subscriptions as well as their value or their cardinality, so it is impossible to re-create the same environment. Secondly, we were not able to find where to download E-StreamHub and thus we could not run it within our environment. Nonetheless, since our aim is not to show that our system is faster that E-StreamHub in some environment but that state repartitioning can be achieved with reasonable performance, we do not think the lack of a direct comparison is a blocking point.

At first, we tested throughput performance with different configuration deployments to find out where the processing time was dominated by matching time instead of network time. We started our tests with a 1-1-1 configuration, that is, one access point, one matcher and one exit point with the addition of one connection point bound to the access point. Before measuring performance, we created $100 \mathrm{k}$ notifications and one million subscriptions in order to assure that object creation time did not affect the measured throughput. In addition, the notifications were created to match at least one subscription in order to be able to constantly measure notification throughput.

This is a worst-case scenario setup since the system has to deliver all the notifications, whereas, in a real environment, some notifications will not be delivered because no matching subscriptions will have been sent. As shown in Figure 7, when E-SilboPS was deployed with a 1-1-1 configuration, notification throughput started to slow down just after the system was loaded with $10 \mathrm{k}$ subscriptions. It descended at a constant rate, and processing time started to be dominated by matching as of $20 \mathrm{k}$ subscriptions. This means that the constant throughput on the left side of the graph is due to network saturation. This limits the amount of messages that an operator can process [31].

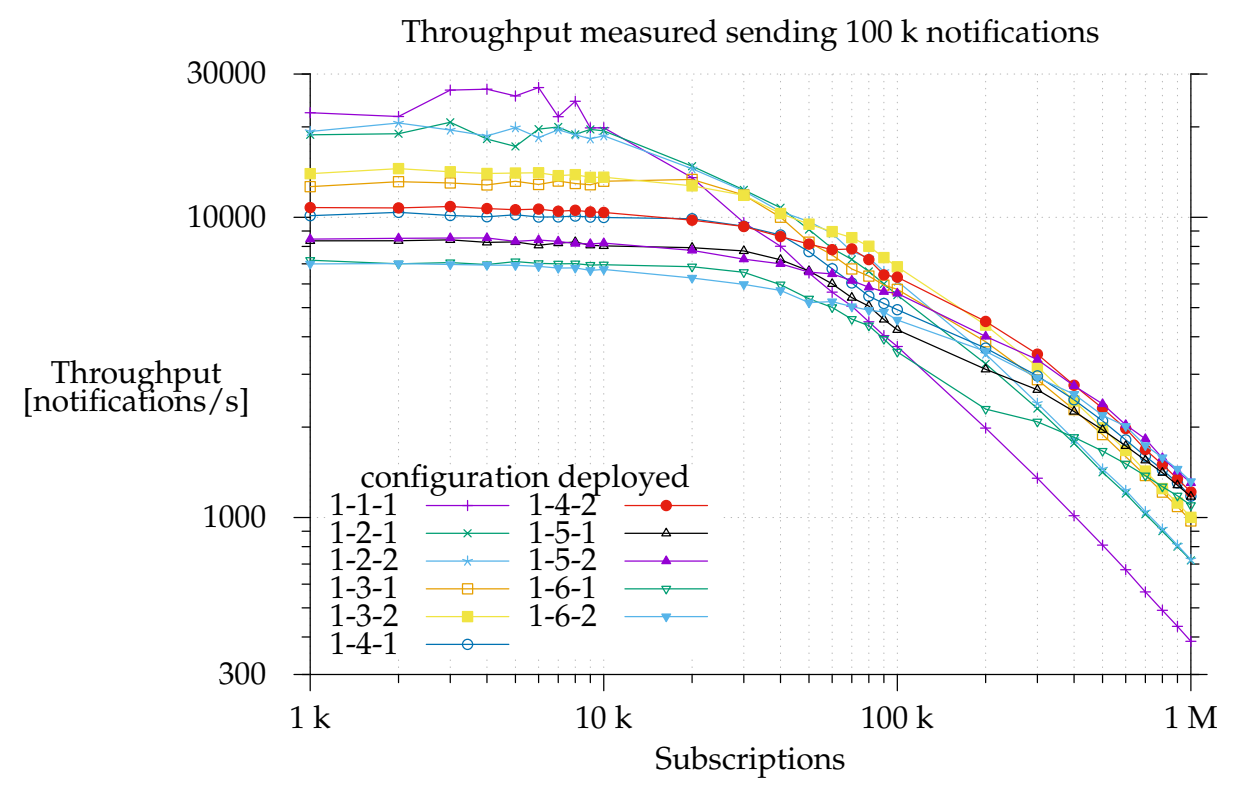

Figure 7. Notification throughput with different configurations: the triplet in the legend represents the number of instances deployed for Access Point, Matcher and Exit Point. 
This is proven by the fact that there is a decrease in notification throughput when the number of operators, and consequently the number of messages passing to the network layer, increases, but the workload is unchanged. Unsurprisingly, minimum notification throughput is observed with the configuration deployment that has the maximum number of elements (1-6-2). In the same way, we find that increasing the numbers of matchers on the right side of the graph, where the matching time is dominant, improves throughput, because, on a per operator basis, the state is smaller, and thus it takes less time to calculate the partial set of subscribers. This is really important since it proves that the current architecture is able to scale out, which is a prerequisite for elasticity [24].

Another interesting point is that the addition of more matchers not only increases the throughput but also changes the slope of the curve. This is consistent with the region in the center of the graph where each matcher is working. If there are fewer subscriptions, the processing time is divided more equally between network time and matching time, and, ideally, this division will be such that the two are balanced leading to $100 \%$ usage of both resources [26,31].

Note also that it is in the region between $50 \mathrm{k}$ and $300 \mathrm{k}$ subscriptions where performance differences between configurations that have only one exit point and others that have two start to appear. As a matter of fact, the 1-x-2 configuration always outperforms the 1-x-1 configuration, and the difference is greater the more matchers it has, as shown in Figure 8.

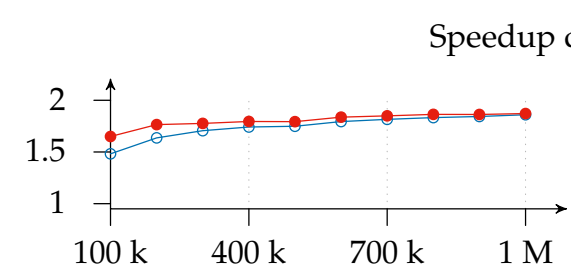

(a)

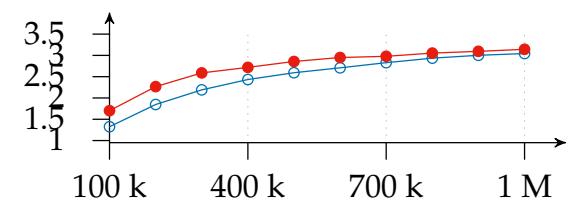

(c)

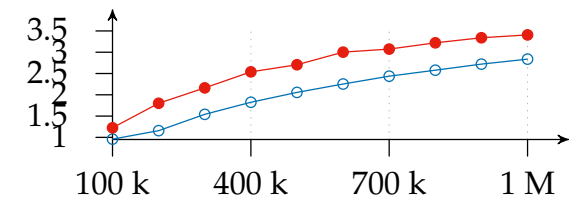

(e)

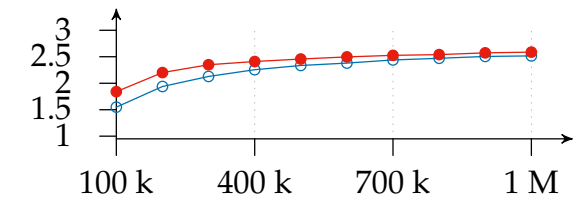

(b)

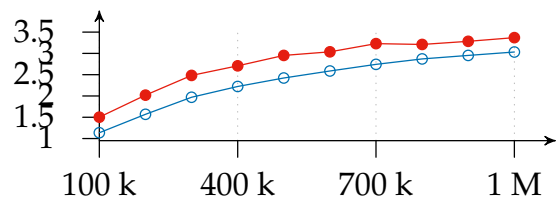

(d)

Figure 8. Notification throughput speedup dominance of 1-x-2 over 1-x-1: the line with filled circles plots the speedup for the 1- $x-2$ configuration, whereas the line with empty circles plots the speedup for the 1-x-1 configuration; plots (a-e) represent matcher instances, namely $x$, scaling from 2 to 6 .

This means that the Exit Point layer does a good job of offloading work from matchers in this region, namely by reducing the partial sets of subscribers to a single set. On the contrary, network saturation limits the total throughput on the left of the graph, hiding any potential gain, whereas the matchers are the factor limiting notification throughput on the right.

Finally, note for Figure 7 that, on the far right, the speedup gained by adding more and more matchers is downward, which is sub-linear. This applies to total efficiency, as shown in Figures 9-11 too. This is consistent with Amdahl's law, Gustafson's law [32,33] and Eager [26]. Even so, the notification throughput provided using the 1-6-2 was 3.4 times greater than using the 1-1-1 configuration.

Figure 10 is useful for cost-benefit analysis of the scaling, since it shows where, for a given configuration deployment and load, it is worth adding another matcher. We find that the top speedup for a $100 \mathrm{k}$ load is achieved with a 1-3-2 configuration. This means that adding more resources simply 
results in a worse throughput because of message overhead. Similarly, a workload increase pushes the curve's peak to the right of the graph.

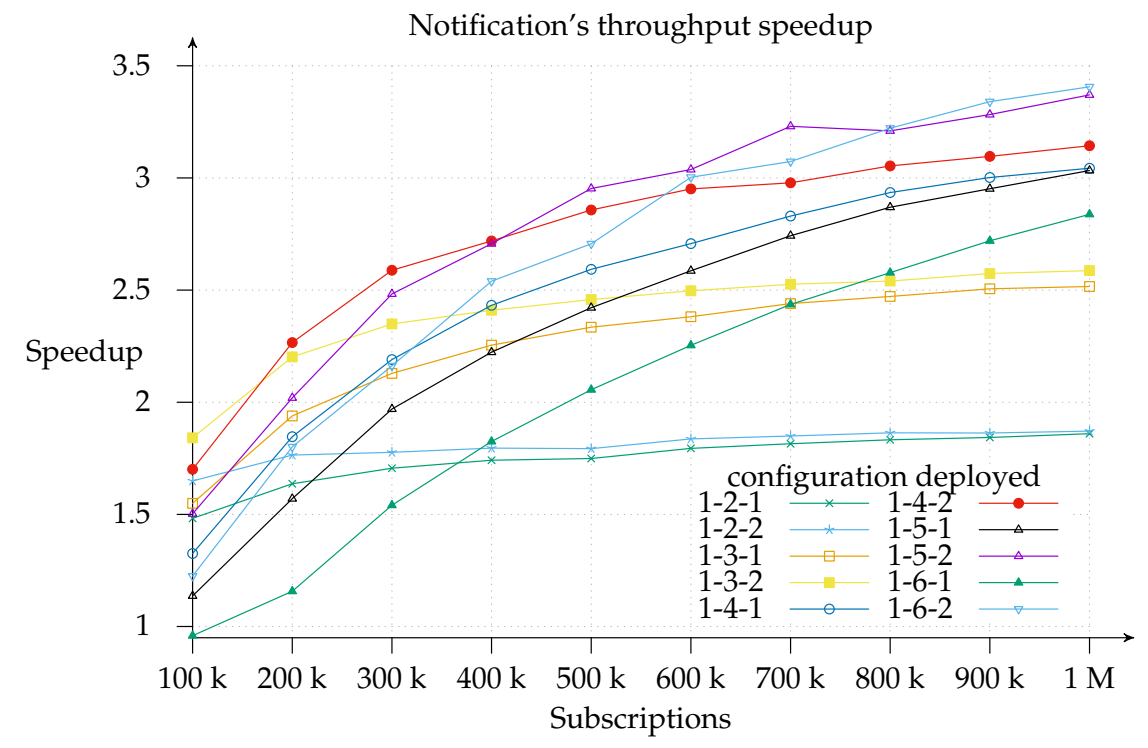

Figure 9. Notification throughput speedup for different configurations with an increasing subscriptions load.

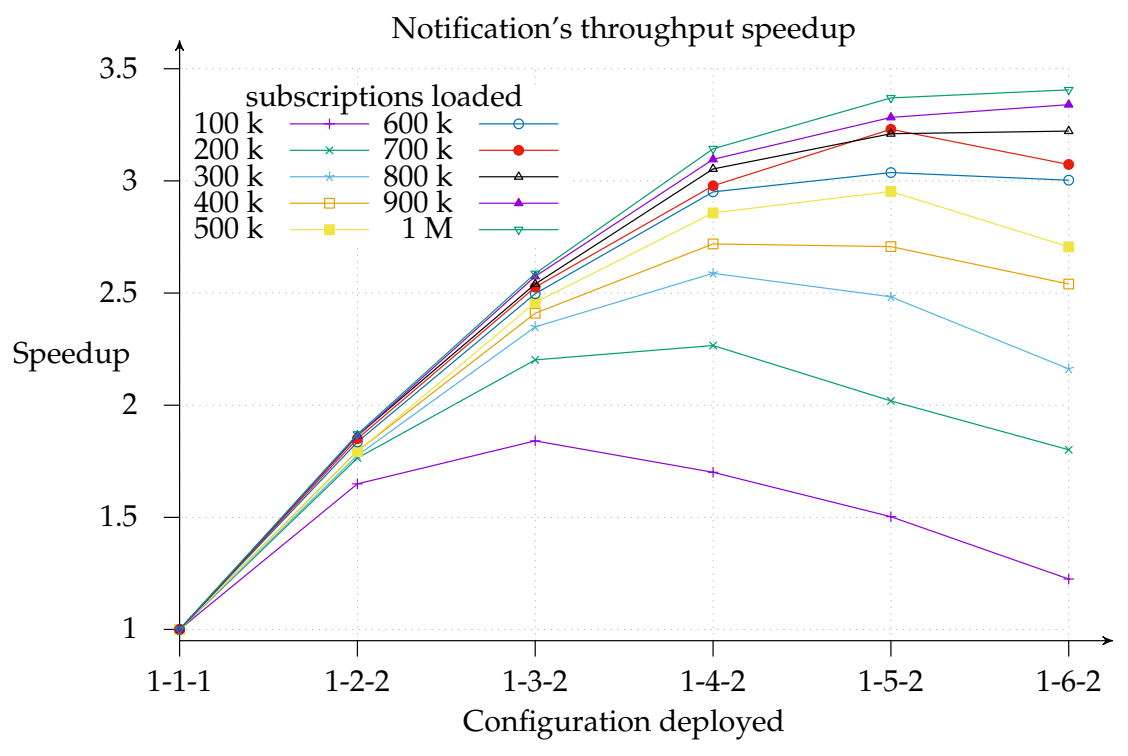

Figure 10. Notification throughput speedup sliced on 1-x-2 configurations.

For clarity's sake, Figure 10 shows the speedup for 1-x-2 deployments only, since, as shown in Figure 8 above, the 1-x-2 configuration always outperforms 1-x-1. However, the same does not apply with respect to efficiency, which is computed as a ratio of speedup to the number of instances (processors) used, that is, $E(n)=\frac{S(n)}{n}$ [26]. Figure 11 shows how efficiency decreases as the number of slices increases and the 1-x-1 configuration is more efficient than the 1-x-2 configuration in most cases and especially with deployments with not many instances. Additionally, this graph indicates the layer to which it is better to add a new slice since deployments with the same number of slices (highlighted regions) can be compared and a cost-benefit analysis of adding a second exit point (white regions) can be conducted. As a matter of fact, we find that the most efficient deployment for the three-slice region for all loads except $100 \mathrm{k}$ is the configuration with only one exit point. This means that matcher requirements are greater than exit point utility. The same applies for the four-slice region, although the 
threshold is raised to a $400 \mathrm{k}$ subscriptions load. This is consistent with expectations since the speedup contribution margin of each new added matcher will be smaller than its predecessor's. This could be modeled in an open cloud using the Shapley value $[34,35]$.

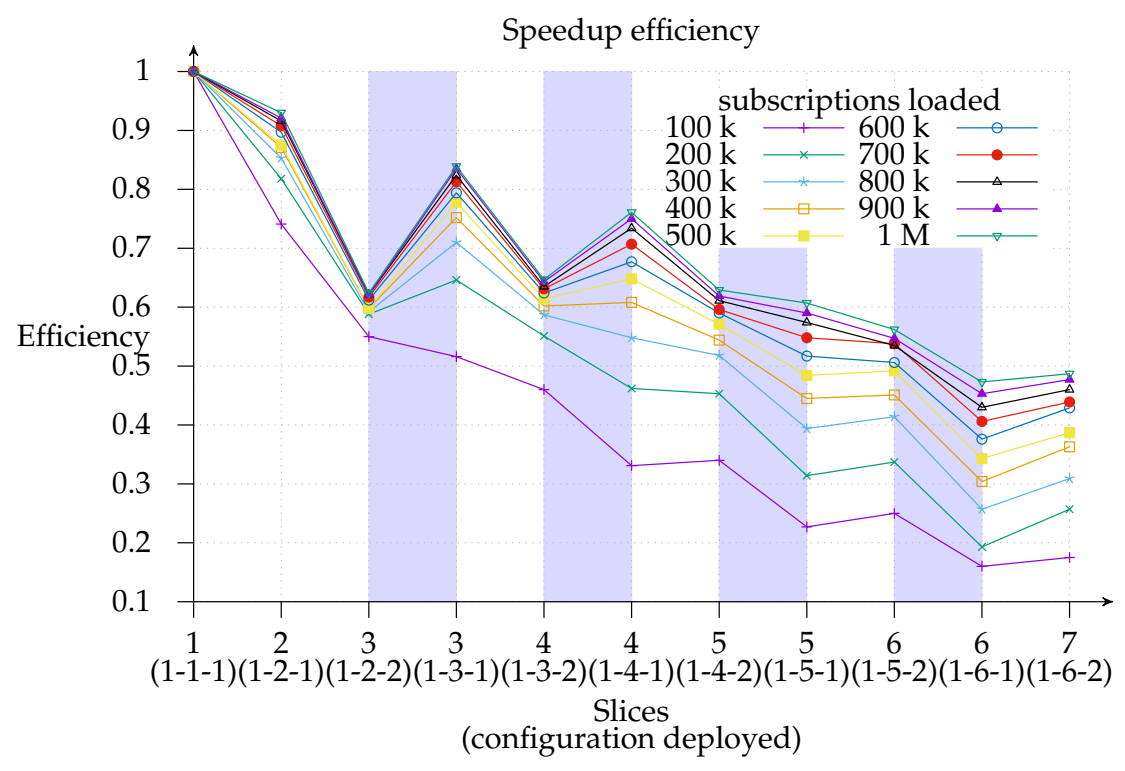

Figure 11. Notification throughput speedup efficiency: a horizontal line means equal efficiency, the highlighted region represents an equal number of slices where a horizontal line also denotes an equal speedup, whereas equal speedup is represented by the local hyperbola in the white region.

Likewise, we observe that adding an exit point in the five- and six-matcher region will help to improve both efficiency and speedup: this suggests that it is better to add an exit point than a matcher in such deployments. Thus, we can infer, as a rule of thumb, that one exit point is necessary for every four matcher instances, irrespective of the number of loaded subscriptions.

Finally, Figure 12 shows the estimated Karp-Flatt metric $e=\frac{\frac{1}{s}-\frac{1}{p}}{1-\frac{1}{p}}$ of configuration deployments, where $s$ represents the speedup and $p$ the number of slices (processors) used. This metric is used to estimate the serial part and the overhead from experimental data [36]. It is, therefore, a valuable metric for use in conjunction with efficiency, since it gives an insight into whether the decreased efficiency is due to the limits of parallelism or overhead. As we can see, we are experiencing network overhead with a $100 \mathrm{k}$ load since adding more matchers increases the estimated $e$, especially with the 1-x-1 configuration. Looking at how the same deployment behaves with a larger subscriptions load, we find proof that the overhead is network related: the estimated $e$ sinks to the $10-20 \%$ region.

In order to measure system behavior during scale-out, we loaded the system with $100 \mathrm{k}$ subscriptions and set up a constant stream of 2000 notifications/s and 1000 subscriptions/s; these are the orders of magnitude normally used by publish subscribe systems like SIENA, PADRES and others to evaluate how the prototype performs under load, they are related to the machine used. Thus, we can examine the throughput of notifications delivered to the subscriber to see how the different Matcher layer scale-out phases affect the throughput of notifications. We ran this test with all deployments, finding that all the results were very similar.

Figure 13 shows the scale-out from the 1-1-1 to the 1-3-1 configuration. The red line represents notification throughput received from the subscriber and the highlighted blue region represents the time during which the system was scaling out. Clearly, the system continues to process notifications while the subscriptions are buffered in the access points and matchers are switching to their new state, after which the access point starts buffering notifications and flushes all its buffered subscriptions (green region). This phase is illustrated in the graph from seconds 11.4 to $11.9(490 \mathrm{~ms})$. The matchers then have their 
new state updated so that they can reprocess the notifications. During the last phase (narrow orange band), which takes place from seconds 11.9 to 12.0 (98 ms), all notifications, buffered while sending the previously buffered subscriptions, are flushed. Obviously, since each matcher operates independently of the others, throughput is highly variable. The normal throughput of 2000 notifications/s is restored at the end of the scale-out, meaning that all internal buffers have been emptied.

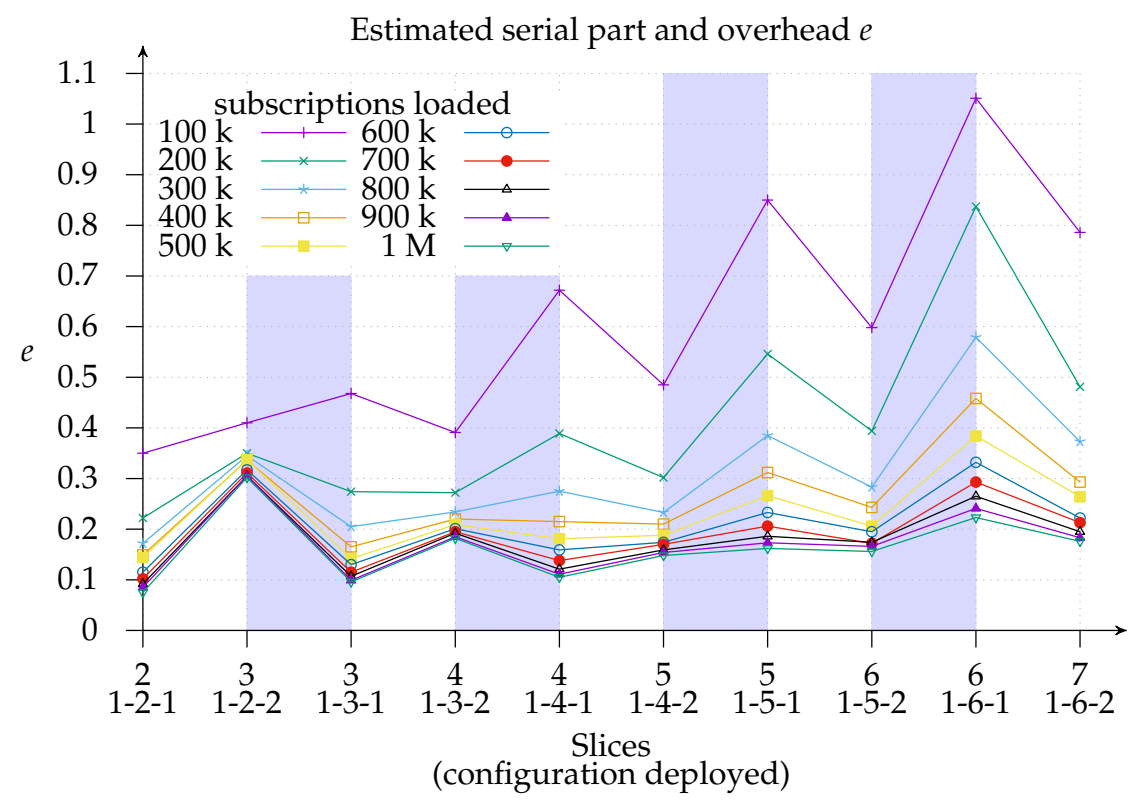

Figure 12. Estimation $e$ of the serial part and overhead using the Karp-Flatt metric: the smallest load (100 k) clearly yields a larger overhead, whereas the biggest load (1 M) reduces overhead to $10 \%$. This reduction is present in all configurations deployed and is directly proportional to the number of matchers. However, the number of exit points has a positive effect in that it lowers $e$. Therefore, the estimation of $e$ can be attributed to network overhead rather than to the intrinsic serial part of matching notifications.

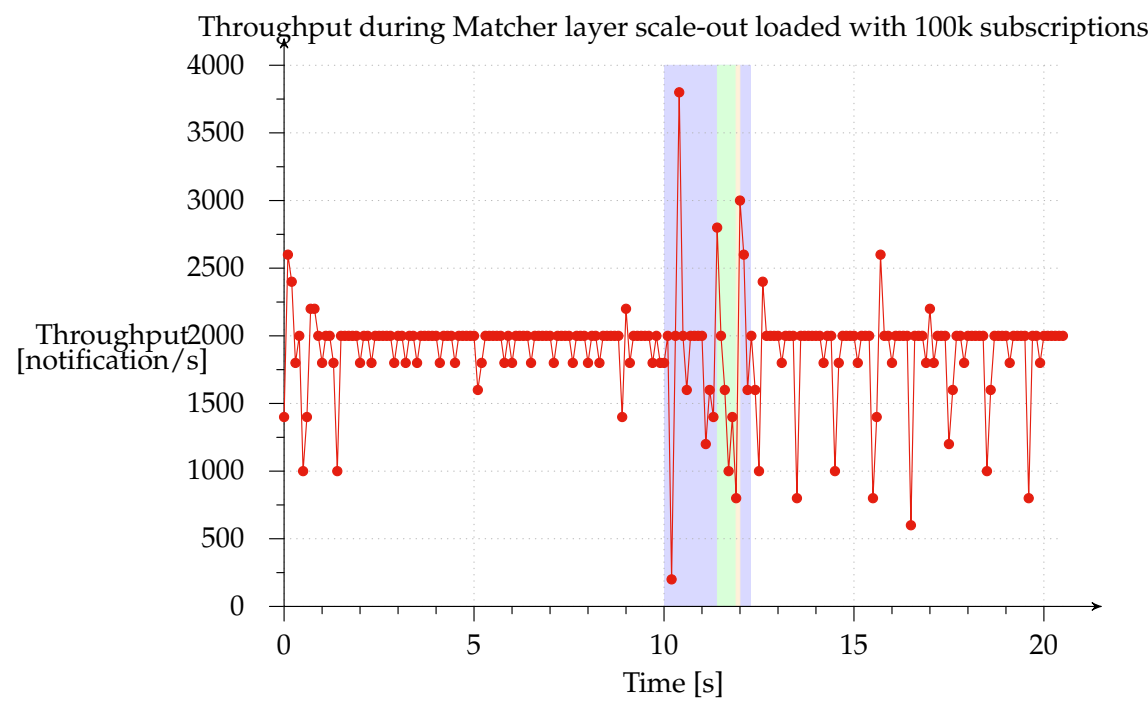

Figure 13. Notification throughput with subscriptions during scale-out of Matcher layer: the deployed system configuration was 1-1-1, scaling out to 1-3-1 and pre-loaded with $100 \mathrm{k}$ subscriptions. The red line is the notification throughput delivered to subscribers while publishing 2000 notifications/s and adding 1000 subscriptions/s. 
We found that notification and subscription throughput has a linear impact on the duration of the scaling window. However, the system always completes the scale-out in a finite amount of time since the size of the buffers that it has to empty is determined by the time taken to complete each phase and the buffers are not updated with new subscriptions or notifications once a phase is complete. In the test that we performed, the time taken to scale out the system ranged from 1 to $8 \mathrm{~s}$ with notification and subscription throughput ranging from 1000 to 8000 messages per second.

This result shows how dynamic state partitioning can be achieved in an acceptable amount of time, thereby solving the problem of forecasting the maximum number of slices required during peak loads and providing the system with the most efficient configuration for the actual load. This is the major contribution of our research on implementing a proper elastic content-based publish/subscribe system. It differs from other systems [17] that use pre-state partitioning and simply move slices around. These systems offer no more than application-specific optimized versions of the VM migration provided by all major hypervisors today.

\section{Future Work}

E-SilboPS currently depends on an external system to decide when it has to scale in or out. This system has to make this decision based on current resource usage, as well as delivered performance. The use of metrics that measure only CPU or memory usage is not very suitable for a cloud system because they are only helpful for the technicians that manage the cloud. Users neither know nor care about how much CPU or how many machines are used, they are concerned about service quality such as Service Level Agreement (SLA), their user experience and billing cost. This means that the software that decides when to scale E-SilboPS in or out should have access to the above metrics and should possibly predict peaks of usage with respect to the SLA. At the same time, it should have knowledge of the performance efficiency and monetary cost of the current configuration, which should be optimum [37-42].

With E-SilboPS, we were able to add elasticity to a content-based publish/subscribe system. One possible improvement of our current model is to offer slice replacement. As a matter of fact, E-SilboPS manages slices of the same operator as a stack. This means that, in order to remove a specific machine, the system needs should scale in until that slice is no longer used. On the other hand, a better approach for this kind of operation is to use the VM migration offered by today's hypervisors: thus, the VM containing the slice can be moved without any system scaling.

A second improvement is to take full advantage of the availability of network multicast: this will reduce the number of messages to be sent and CPU usage. This is warranted thorough validation since it could perform as well as the current implementation in a virtualized environment, where, unlike high-end routers, there are no physical processors dedicated to each endpoint.

Another potentially interesting feature deserving support is multilayer scalability: the capacity to scale all three layers in and out independently of each other within a single scaling transaction. However, this should be weighted against the increased complexity of the algorithm. Apart from in-field validation of the use case, we also need to check that it delivers the expected improvements or instead merely increases software complexity.

The last feature that we think would add value to the system is the capability to handover connections between connection points without disconnecting clients: this feature would enable load balancing by repartitioning connections looking at producer or consumer throughput.

Future work will be carried out within a number of use cases of the IoF2020 Horizon 2020 project [43], such as the Poultry Chain Management [44] one, in which we will deploy our proposed architecture in a distributed environment to collect/monitor a huge amount of data coming from three critical points in the poultry chain (i.e., at the farm, during transport, and in the slaughter house), to later provide daily insights and early warnings based on the available data. This scenario will allow us to test how E-SilboPS performs in a real large-scale IoT scenario, handling big data, and being connected through different networks with varying speed and latency. 


\section{Conclusions}

In this article, we have presented a proper elastic content-based publish/subscribe system that supports transparent, publisher-wise dynamic state repartitioning without client disconnection and with minimal notification delivery interruption for subscribers.

As shown in the evaluation section, E-SilboPS can be successfully used as communication mechanism between IoT devices and any kind of application interested in the data generated by them that implements the proposed general architecture. Moreover, thanks to its elastically-scalable nature, it can sustain the large amount of data that today's IoT services can generate.

Unlike other proposals such as [16], which focuses on fault tolerance and thus requires more space to store the redundant state and also a more complex algorithm for execution by the Access Point layer, or [17], which is really a reimplementation of an application-specific version of the VM migration capability offered by today's hypervisors, we described the full algorithm that we validated with qualitative and quantitative measurements. This demonstrated that dynamic state repartitioning is not only possible but also achievable even from the performance point of view.

Finally, note that the reported research is being developed and applied as part of the 4CaaSt, FIWARE and FICORE EU FP7 projects, where Publish/Subscribe is offered as both a value-added service to hosted applications and a key internal platform asset.

Acknowledgments: The research leading to these results was partially funded by the European Commission Seventh Framework Programme (FP7 and the H2020 program) 4CaaSt, FIWARE, FICORE and FINEXT projects (https://www.fiware.org/) under Grant Nos. 258862, 285248, 632893 and 732851. This article expresses the opinions of the authors and not necessarily those of the European Commission. The European Commission is not liable for any use that may be made of the information contained in this paper.

Author Contributions: Sergio Vavassori conceived and designed the system and the algorithm; Javier Soriano contributed the general architecture and made significant contributions to both the system concept and the algorithm during their refinement; Rafael Fernandez conceived and designed the experiment, performed the experiment and analyzed the data; all the coauthors contributed substantially to writing and reviewing the paper.

Conflicts of Interest: The authors declare no conflict of interest.

\section{References}

1. Memos, V.A.; Psannis, K.E.; Ishibashi, Y.; Kim, B.G.; Gupta, B. An Efficient Algorithm for Media-based Surveillance System (EAMSuS) in IoT Smart City Framework. Future Gener. Comput. Syst. 2017, (in press).

2. Laleh, T.; Mokhov, S.A.; Paquet, J.; Yan, Y. Context-Aware Cloud Service Brokerage: A Solution to the Problem of Data Integration among SaaS Providers. In Proceedings of the Eighth International C* Conference on Computer Science \& Software Engineering, C3S2E '15, Yokohama, Japan, 13-15 July 2015; ACM: New York, NY, USA, 2008; pp. 46-55.

3. OpenStack. Available online: https://www.openstack.org/ (accessed on 6 September 2017).

4. OpenNebula. Available online: https://opennebula.org/ (accessed on 6 September 2017).

5. An, K.; Pradhan, S.; Caglar, F.; Gokhale, A. A Publish/Subscribe Middleware for Dependable and Real-time Resource Monitoring in the Cloud. In Proceedings of the Workshop on Secure and Dependable Middleware for Cloud Monitoring and Management (SDMCMM '12), Montreal, QC, Canada, 3-5 December 2012; ACM: New York, NY, USA, 2012; pp. 3:1-3:6.

6. Pavloski, M.; Gelenbe, E. Mitigating for Signalling Attacks in UMTS Networks. In Proceedings of the 29th International Symposium on Computer and Information Sciences, Information Sciences and Systems 2014-ISCIS 2014, Krakow, Poland, 27-28 October 2014; pp. 159-165.

7. Alhamazani, K.; Ranjan, R.; Mitra, K.; Rabhi, F.A.; Jayaraman, P.P.; Khan, S.U.; Guabtni, A.; Bhatnagar, V. An overview of the commercial cloud monitoring tools: Research dimensions, design issues, and state-of-the-art. Computing 2015, 97, 357-377.

8. Google Pub/Sub. Available online: https://aws.amazon.com/sns/ (accessed on 6 September 2017).

9. AWS Simple Notification Service. Available online: https://cloud.google.com/pubsub/docs/overview (accessed on 6 September 2017). 
10. Microsoft Azure Service Bus Messaging. Available online: https://azure.microsoft.com/en-us/ documentation/articles/service-bus-messaging-overview (accessed on 6 September 2017).

11. Mühl, G.; Fiege, L.; Pietzuch, P. Distributed Event-Based Systems; Springer: Secaucus, NJ, USA, 2006.

12. Shen, H. Content-Based Publish/Subscribe Systems. In Handbook of Peer-to-Peer Networking; Shen, X., Yu, H., Buford, J., Akon, M., Eds.; Springer: Boston, MA, USA, 2010; pp. 1333-1366.

13. Carzaniga, A.; Wolf, A.L. Forwarding in a content-based network. In Proceedings of the 2003 Conference on Applications, Technologies, Architectures, and Protocols for Computer Communications (SIGCOMM '03), Karlsruhe, Germany, 25-29 August 2003; ACM: New York, NY, USA, 2003; pp. 163-174.

14. Barazzutti, R.; Felber, P.; Fetzer, C.; Onica, E.; Pineau, J.F.; Pasin, M.; Rivière, E.; Weigert, S. StreamHub: A Massively Parallel Architecture for High-performance Content-based Publish/Subscribe. In Proceedings of the 7th ACM International Conference on Distributed Event-based Systems (DEBS '13), Arlington, TX, USA, 29 June-3 July 2013; ACM: New York, NY, USA, 2013; pp. 63-74.

15. Ma, X.; Wang, Y.; Qiu, Q.; Sun, W.; Pei, X. Scalable and elastic event matching for attribute-based publish/subscribe systems. Future Gener. Comput. Syst. 2014, 36, 102-119.

16. Li, M.; Ye, F.; Kim, M.; Chen, H.; Lei, H. A Scalable and Elastic Publish/Subscribe Service. In Proceedings of the 2011 IEEE International Parallel Distributed Processing Symposium (IPDPS), Anchorage, AK, USA, 16-20 May 2011; pp. 1254-1265.

17. Barazzutti, R.; Heinze, T.; Martin, A.; Onica, E.; Felber, P.; Fetzer, C.; Jerzak, Z.; Pasin, M.; Rivière, E. Elastic Scaling of a High-Throughput Content-Based Publish/Subscribe Engine. In Proceedings of the 2014 IEEE 34th International Conference on Distributed Computing Systems (ICDCS '14), Madrid, Spain, 30 June-3 July 2014; IEEE Computer Society: Washington, DC, USA, 2014; pp. 567-576.

18. Vavassori, S.; Soriano, J.; Lizcano, D.; Jiménez, M. Explicit Context Matching in Content-Based Publish/Subscribe Systems. Sensors 2013, 13, 2945-2966.

19. FIWARE Internet of Things (IoT) Services Enablement Architecture. Available online: https: / / forge.fiware.org/plugins/mediawiki/wiki/fiware/index.php/Internet_of_Things_\%28IoT\%29_Services_ Enablement_Architecture (accessed on 6 September 2017).

20. NGSI-9/NGSI-10 Information Model. Available online: https://forge.fiware.org/plugins/mediawiki/wiki/ fiware/index.php/NGSI-9/NGSI-10_information_model (accessed on 6 September 2017).

21. FIWARE NGSI Open RESTful API Specification. Available online: https://forge.fiware.org/plugins/ mediawiki/wiki/fiware/index.php/FI-WARE_NGSI_Open_RESTful_API_Specification (accessed on 6 September 2017).

22. Mashup FIWARE Lab: Madrid Air Quality. Available online: https://mashup.lab.fiware.org/testuser/ madrid-air-quality (accessed on 6 September 2017).

23. Esposito, C.; Castiglione, A.; Palmieri, F.; Ficco, M.; Choo, K.K.R. A Publish/Subscribe Protocol for Event-Driven Communications in the Internet of Things. In Proceedings of the 2016 IEEE 14th International Conference on Dependable, Autonomic and Secure Computing, 14th International Conference on Pervasive Intelligence and Computing, 2nd International Conference on Big Data Intelligence and Computing and Cyber Science and Technology Congress (DASC/PiCom/DataCom/CyberSciTech), Auckland, New Zealand, 8-12 August 2016; pp. 376-383.

24. Herbst, N.R.; Kounev, S.; Reussner, R. Elasticity in Cloud Computing: What It Is, and What It Is Not. In Proceedings of the 10th International Conference on Autonomic Computing (ICAC 13), San Jose, CA, USA, 26-28 June 2013; USENIX: San Jose, CA, USA, 2013; pp. 23-27.

25. Stoica, I.; Morris, R.; Karger, D.; Kaashoek, M.F.; Balakrishnan, H. Chord: A Scalable Peer-to-peer Lookup Service for Internet Applications. In Proceedings of the 2001 Conference on Applications, Technologies, Architectures, and Protocols for Computer Communications (SIGCOMM '01), San Diego, CA, USA, 27-31 August 2001; ACM: New York, NY, USA, 2001; pp. 149-160.

26. Eager, D.L.; Zahorjan, J.; Lozowska, E.D. Speedup Versus Efficiency in Parallel Systems. IEEE Trans. Comput. 1989, 38, 408-423.

27. Apache ZooKeeper. Available online: https://zookeeper.apache.org/ (accessed on 6 September 2017).

28. Lehrig, S.; Eikerling, H.; Becker, S. Scalability, Elasticity, and Efficiency in Cloud Computing: A Systematic Literature Review of Definitions and Metrics. In Proceedings of the 11th International ACM SIGSOFT Conference on Quality of Software Architectures (QoSA '15), Montreal, QC, Canada, 4-8 May 2015; ACM: New York, NY, USA, 2015; pp. 83-92. 
29. Chandy, K.M.; Lamport, L. Distributed Snapshots: Determining Global States of Distributed Systems. ACM Trans. Comput. Syst. 1985, 3, 63-75.

30. SilboPS. Available online: https://github.com/conwetlab/silbops (accessed on 6 September 2017).

31. Lazowska, E.D.; Zahorjan, J.; Graham, G.S.; Sevcik, K.C. Quantitative System Performance: Computer System Analysis Using Queueing Network Models; Prentice-Hall, Inc.: Upper Saddle River, NJ, USA, 1984.

32. Amdahl, G.M. Validity of the Single Processor Approach to Achieving Large Scale Computing Capabilities. In Proceedings of the Spring Joint Computer Conference ( AFIPS '67 (Spring)), Atlantic City, NJ, USA, 18-20 April 1967; ACM: New York, NY, USA, 1967; pp. 483-485.

33. Gustafson, J.L. Reevaluating Amdahl's Law. Commun. ACM 1988, 31, 532-533.

34. Shapley, L.S. A value for n-person games. Contribution to the Theory of Games. Ann. Math. Stud. 1953, $2,28$.

35. Roth, A.E. The Shapley Value: Essays in Honor of Lloyd S. Shapley; Cambridge University Press: Cambridge, UK, 1988.

36. Karp, A.H.; Flatt, H.P. Measuring Parallel Processor Performance. Commun. ACM 1990, 33, 539-543.

37. Mao, M.; Humphrey, M. Auto-scaling to Minimize Cost and Meet Application Deadlines in Cloud Workflows. In Proceedings of the 2011 International Conference for High Performance Computing, Networking, Storage and Analysis (SC '11), Seatle, WA, USA, 12-18 November 2011; ACM: New York, NY, USA, 2011; pp. 49:1-49:12.

38. Mao, M.; Humphrey, M. A Performance Study on the VM Startup Time in the Cloud. In Proceedings of the 2012 IEEE Fifth International Conference on Cloud Computing (CLOUD '12), Honolulu, HI, USA, 24-29 June 2012; IEEE Computer Society: Washington, DC, USA, 2012; pp. 423-430.

39. Mao, M.; Humphrey, M. Scaling and Scheduling to Maximize Application Performance within Budget Constraints in Cloud Workflows. In Proceedings of the 2013 IEEE 27th International Symposium on Parallel and Distributed Processing (IPDPS '13), Boston, MA, USA, 20-24 May 2013; IEEE Computer Society: Washington, DC, USA, 2013; pp. 67-78.

40. Galloway, J.M.; Smith, K.L.; Vrbsky, S.S. Power aware load balancing for cloud computing. In Proceedings of the World Congress on Engineering and Computer Science, San Francisco, CA, USA, 19-21 October 2011; Volume 1, pp. 19-21.

41. Galloway, J.M.; Smith, K.; Carver, J. An Empirical Study of Power Aware Load Balancing in Local Cloud Architectures. In Proceedings of the 2012 Ninth International Conference on Information Technology-New Generations (ITNG '12), Las Vegas, NV, USA, 16-18 April 2012; IEEE Computer Society: Washington, DC, USA, 2012; pp. 232-236.

42. Nguyen, P.; Nahrstedt, K. Resource Management for Elastic Publish Subscribe Systems: A Performance Modeling-Based Approach. In Proceedings of the 2016 IEEE 9th International Conference on Cloud Computing (CLOUD), San Francisco, CA, USA, 27 June-2 July 2016; pp. 561-568.

43. Internet of Food \& Farm 2020, an European Union's Horizon 2020 Research and Innovation Programme under Grant Agreement 731884. Available online: https: / www.iof2020.eu/ (accessed on 6 September 2017).

44. Poultry Chain Management Use Case of IoF2020. Available online: https://www.iof2020.eu/trials/meat/ poultry-chain-management (accessed on 6 September 2017).

(C) 2017 by the authors. Licensee MDPI, Basel, Switzerland. This article is an open access article distributed under the terms and conditions of the Creative Commons Attribution (CC BY) license (http://creativecommons.org/licenses/by/4.0/). 